\title{
Modelling molecular transformations in ferroelectric polymers induced by mechanical and electrical means
}

\author{
H.M.G. CORREIA ${ }^{\mathrm{a}}$, AND M.M. D.RAMOS ${ }^{\mathrm{a}}$ \\ ${ }^{a}$ Departamento de Física, Universidade do Minho, \\ Campus de Gualtar, 4710-057 Braga, Portugal
}

We describe a new approach to model the dynamical structural modifications of ferroelectric polymer molecules induced by uniaxial stretching and by the application of electric fields of various strengths and orientations. Our approach combines a self-consistent quantum mechanical method with molecular dynamics, removing the need to use inter-atomic potentials in the dynamic calculations. Here we present results obtained for individual molecules of poly(vinylidene fluoride) in its alpha and beta form. The effects of structural disorder due to the presence of inverted monomer units in a "headto-head" and "tail-to-tail" position are also discussed.

Keywords: Atomistic modeling; Monomer inversion; Poling; Stretching

\section{INTRODUCTION}

Polyvinylene fluoride (PVDF) is a ferroelectric polymer with piezoelectric and pyroelectric properties suitable to be used in a wide range of applications, including microphones, transducers, sensors and actuators [1]. Molecular dipolar orientation is believed to be one of the mechanisms contributing to these properties. In many of the above mentioned applications, PVDF is subjected to an applied electric field for a specific period of time. Therefore, there is a need for characterizing the response of PVDF to the applied field at molecular level.

It is well established that PVDF is a polymer with three different molecular conformations and five types of crystalline structure, depending on its preparation conditions [2]. While the 
alpha-phase with trans-gauche-trans-gauche chains is the most common one, the beta-phase with zigzag all-trans chains is the most technologically interesting one due to its stronger pyroelectric and piezoelectric properties. The beta-PVDF form can be obtained directly from growth in solution or from the transformation of the trans-gauche-trans-gauche conformation to the all-trans planar conformation induced by mechanical deformation and/or electrical poling.

In general PVDF is produced by free-radical polymerization methods. The high reactivity of fluorinated alkyl radicals is responsible for the occasional addition of reversed monomer units leading to head-to-head and tail-to-tail structural defects. The effects of these constitutional defects on molecular modifications induced by mechanical and electrical means are not well understood and further studies need to be done.

The main goal of this work is to improve the understanding of the structural changes that occur at atomic and molecular scale in alpha-PVDF and beta-PVDF chains during the application of an uniform electrical field, as well as those induced by mechanical stretching in alpha-to-beta-transformation. For this purpose, the results of a self-consistent quantum molecular dynamics study of isolated PVDF chains with and without inverted monomer defects are presented and discussed.

\section{THEORETICAL METHOD AND COMPUTATIONAL DETAILS}

A new method is proposed for the atomistic level characterization of relaxation and transformation processes in isolated PVDF chains induced by external force fields and electric fields. The proposed approach combines self-consistent quantum chemistry with molecular dynamics and is implemented in the CHEMOS code $[3,4]$. This code has further facilities to include external fields (electric and force fields). The quantum chemistry is carried out using a semi-empirical application of the Hartree-Fock theory, called CNDO (Complete Neglect of 
Differential Overlap), to generate analytically self-consistent forces on each atom. The molecular dynamics will then use these forces to move the atoms according to newtonian equations of motion. In this approach there is no need to define inter-atomic potentials in advance. Besides, complicated transformation processes induced by the applied fields can easily emerge from our calculations without pre-knowledge of the mechanism.

In the quantum chemistry calculations, we have used the CNDO/2 parameters proposed by Pople and Beveridge [5], a minimal basis set and a cluster model framework. The polymer system is brought to self-consistency before the atomic relaxation starts and after each molecular dynamics time-step which is approximately $1 \mathrm{fs}$.

Since our previous work [6] has shown that PVDF with alpha and beta conformation have dipole moment and mean polarizability per monomer unit that do not vary significantly with chain length for chains with more than 20 monomer units, we have addressed the interaction of individual $\alpha$ - and $\beta$-PVDF chains with applied electric and force fields by examining the behavior of a $\left[\mathrm{H}-\left(\mathrm{CF}_{2}-\mathrm{CH}_{2}\right)_{20}-\mathrm{H}\right]$ molecule with and without inverted monomer units. Before any interaction with the applied fields is considered, we have relaxed the ground-state geometry of each PVDF chain to equilibrium. The ground-state geometries obtained for defect-free chains are show in figure 1.

(a)

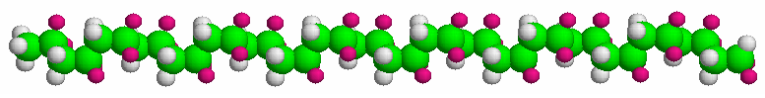

(b)

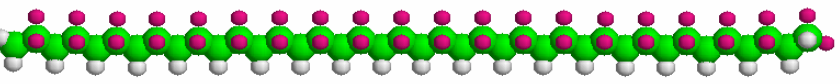

FIGURE 1. The optimized molecular geometry of a defect-free PVDF chain with 20 monomer units and the following molecular conformations: (a) $\alpha$-form; (b) $\beta$-form.

\section{RESULTS AND DISCUSSION}

Since defect-free PVDF chains with alpha and beta conformation have permanent dipole moments, they are expected to be affected by the application of a uniform electric field. The 
effects predicted for an applied electric field with the strength of $5 \mathrm{MV} / \mathrm{cm}$, in a direction that is neither parallel nor perpendicular to the chain, is shown in figure 2 . The main effects predicted for both $\alpha$ - and $\beta$-chains were the rotation of the entire chain around the molecular axis and the bending of the chain in order to align its molecular dipole moment with the applied electric field. These effects are more pronounced for the $\beta$-chain which has the greatest dipole moment [6]. These results are in agreement with our previous work [7].

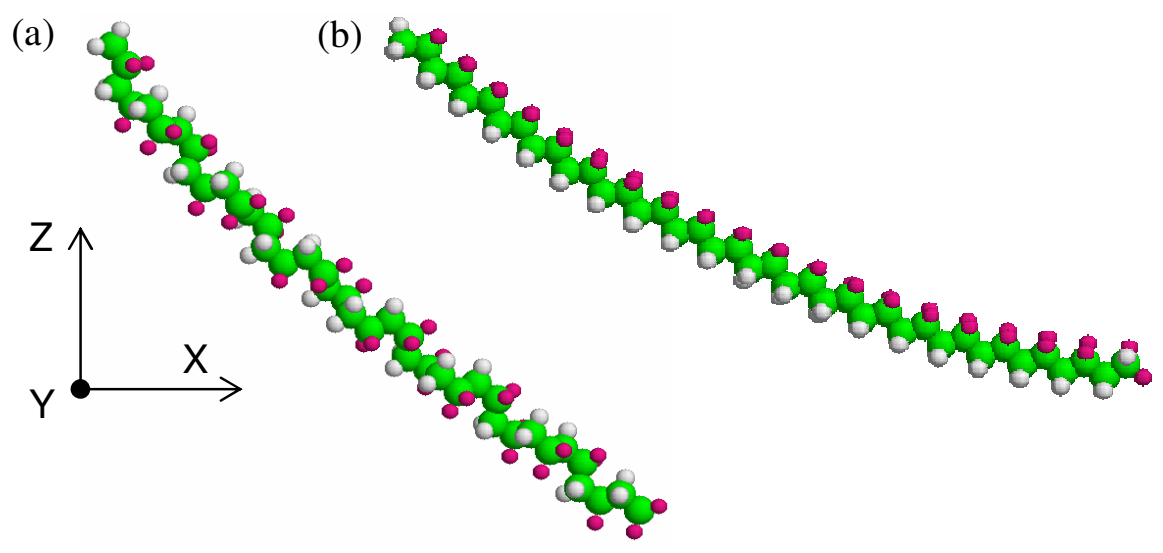

FIGURE 2. The effect of the applied electric field $\vec{E}=(2.9 \hat{i}+2.9 \hat{j}+2.9 \hat{k})(\mathrm{MV} / \mathrm{cm})$ on the defect-free PVDF chain with 20 monomer units: (a) $\alpha$-form; (b) $\beta$-form. The straight polymer chains show in figure 1 were positioned parallel to the $\mathrm{x}$-axis in the absence of the applied field.

When a PVDF chain has one or more inversion monomer units in the centre of the chain, the same applied electric field of $5 \mathrm{MV} / \mathrm{cm}$ leads to the rotation around the molecular axis of the polymer segments with a head-to-tail sequence on both sides of head-to-head and tail-to-tail linkages whereas the $\mathrm{CF}_{2}$ and $\mathrm{CH}_{2}$ dipoles within the inverted monomer units remain unchanged. Furthermore, the amplitude of molecular bending is reduced as the number of inverted monomer units increases. These effects are illustrated in figure 3 for $\beta$-PVDF chains with 1,2 and 3 inverted monomers units in the centre of the chain. The rotation of the molecular segments is principally electrostatic due to the interaction of the dipole moment of the segments with the applied electric field. Moreover, the decrease in molecular bending can 
be explained in terms of a decrease in the molecular dipole moment due to the presence of the constitutional defects $[6,8]$.

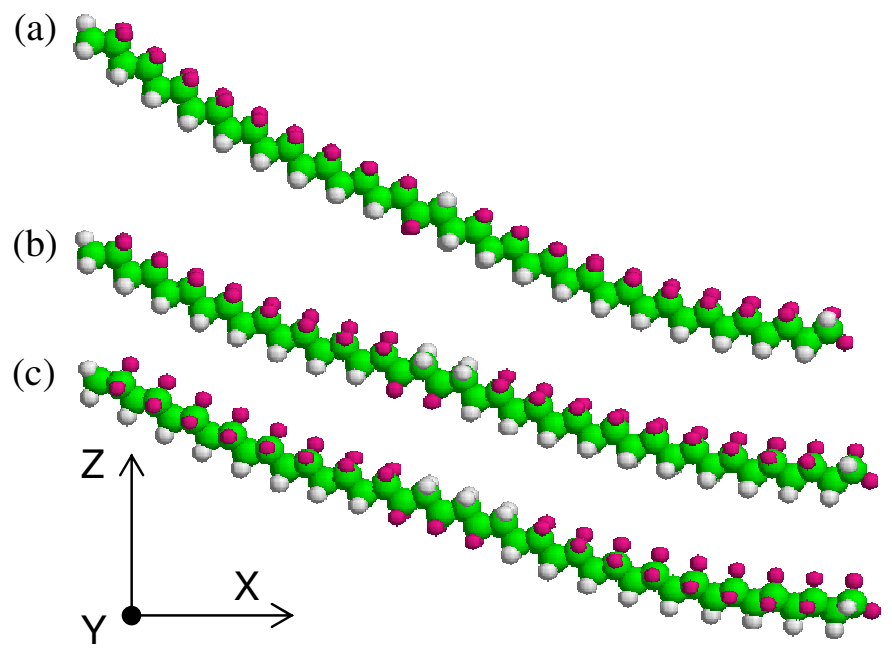

FIGURE 3. The effect of the applied electric field $\vec{E}=(2.9 \hat{i}+2.9 \hat{j}+2.9 \hat{k})(\mathrm{MV} / \mathrm{cm})$ on the $\beta-$ PVDF chain with a single head-to-head and tail-to-tail linkages and the following number of inverted monomer units in the centre of the chain: (a) one; (b) two; (c) three. The straight polymer chains with constitutional defects were positioned parallel to the $\mathrm{x}$-axis in the absence of the applied field.

The calculation was repeated for an electric field twenty times larger and a very different structural modification of the $\alpha$-PVDF chains was obtained. In the case of a defect-free chain, the transformation of the straight $\alpha$-PVDF chains into a nearly straight $\beta$-PVDF chain was predicted when the electric field was applied perpendicular to the chain axis (see figure 4). When the same electric field is applied to the $\alpha$-chain with head-to-head and tail-to-tail defects, nearly straight segments with the $\beta$-form separated by kinks at head-to-head and tailto-tail defects are created within the polymer chain (see figure 5). Since the applied electric field is strong enough, the $\mathrm{CF}_{2}$ and $\mathrm{CH}_{2}$ dipoles show the same orientation in all the segments generated. 


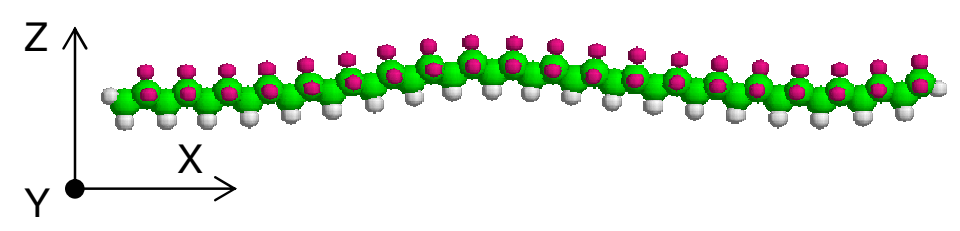

FIGURE 4. The effect of the applied electric field $\vec{E}=(70.7 \hat{j}+70.7 \hat{k})(\mathrm{MV} / \mathrm{cm})$ on a defectfree $\alpha$-PVDF chain with 20 monomer units and the molecular axis aligned with the $\mathrm{x}$-axis in the absence of the applied field.

(a)

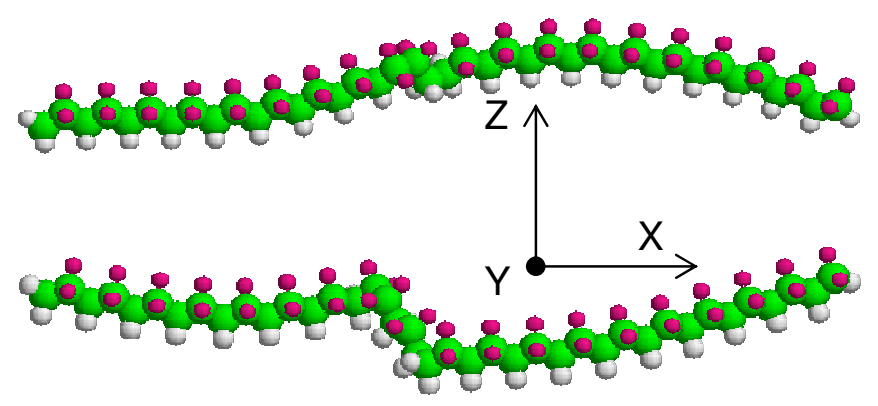

(c)

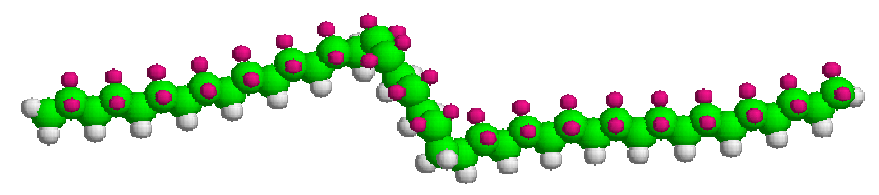

FIGURE 5. The effect of the applied electric field $\vec{E}=(70.7 \hat{j}+70.7 \hat{k})(\mathrm{MV} / \mathrm{cm})$ on the $\alpha-$ PVDF chain with the following number of inverted monomer units in the centre of the chain:

(a) one; (b) two; (c) three. The defective chains were oriented parallel to the x-axis in the absence of the applied field.

As shown in our previous work [7], the transformation of defect-free $\alpha$-PVDF chains with trans-gauche-trans-gauche conformation into $\beta$-type PVDF chains with all-trans zigzag conformation can easily be achieved by mechanical stretching. Alignment of $\mathrm{CF}_{2}$ and $\mathrm{CH}_{2}$ dipoles can also de achieved by applying an electric field perpendicular to the stretched chain. As can be seen in figure 6 , the presence of inverted monomer defects in $\alpha$-PVDF chains does not seem to affect significantly the structural modifications induced by the mechanical stretching and the electrical field on the defect-free parts of the chain. Moreover, the 
transformation of $\alpha$-form to $\beta$-form in the defect region seems to be energetically easier than intra-molecular rotation.

(a)

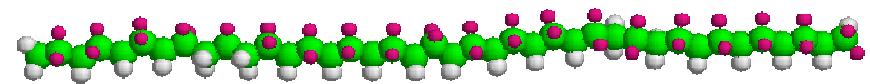

(b)
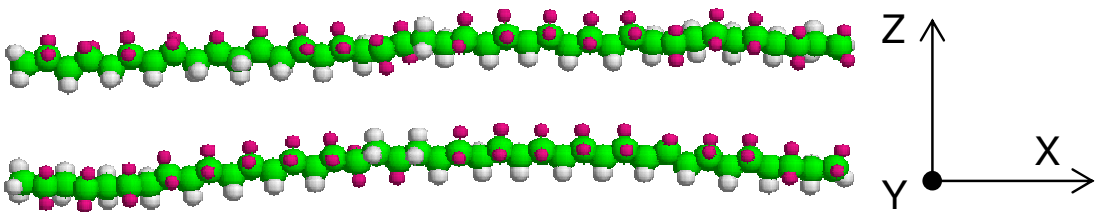

(d)

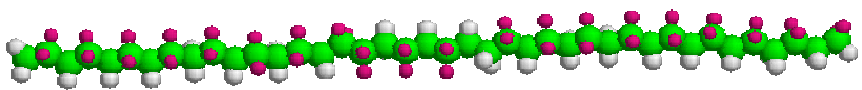

FIGURE 6. The structural modifications of the $\alpha$-PVDF chain without (a) and with (b-d) inverted monomer units induced by the application of a force of $8.24 \mathrm{nN}$ to the carbon atom of both chain ends in opposite directions followed by the application of the electric field $\vec{E}=(35.35 \hat{j}+35.35 \hat{k})(\mathrm{MV} / \mathrm{cm})$. All the chains were oriented parallel to the $\mathrm{x}$-axis in the absence of both force and electric fields.

\section{CONCLUSIONS}

We have studied the structural changes of PVDF chains with all-trans and trans-gauche-transgauche conformations induced by external applied electric fields of different strengths. In addition, the mechanical stretching of $\alpha$-PVDF chains followed by electrical poling was also considered. The main effects predicted were intra-molecular re-orientation and molecular distortion, which are more pronounced for high applied external fields. Our results also suggest that the presence of constitutional defects in the chains, assuming the form of head-tohead and tail-to-tail defects, leads to different dipolar segments within the chain which can be separated by kinks at high applied electric fields.

Although experiments are still ahead of theory, this work offers an exciting prospect of being able to theoretically study the dynamical response of the material at atomic and molecular 
scale. It provides an important starting point for the elucidation of many unanswered questions raised by experiments.

\section{ACKNOWLEDGEMENT}

This work was supported by the Portuguese Foundation for Science and Technology (FCT) and the European Community Fund FEDER under the projects number POCTI/CTM/41574/2001 and CONC-REEQ/443/2001. One of us (H.M.G.C.) is also indebted to FCT for financial support under PhD grant nº. SFRH/BD/11231/2002.

\section{REFERENCES}

[1] Fukada, E.: History and recent progress in piezoelectric polymers. IEEE Transactions on Ultrasonics Ferroelectrics and Frequency Control 47 (6), 1277-1290 (2000).

[2] Nalwa, H.S.: Recent Developments in Ferroelectric Polymers. Journal of Macromolecular Science-Reviews in Macromolecular Chemistry and Physics C31 (4), 341-432 (1991).

[3] Wallace, D.S., Electron-lattice coupling in conjugated polymers, in D. Phil. Thesis. 1989, University of Oxford.

[4] Wallace, D.S., Stoneham, A.M., Hayes, W., Fisher, A.J., and Harker, A.H.: Theory of Defects in Conducting Polymers .1. Theoretical Principles and Simple Applications. Journal of Physics-Condensed Matter 3 (22), 3879-3903 (1991).

[5] Pople, J.A. and Beveridge, D.L.: Approximate Molecular Orbital Theory, New York: McGraw-Hill; 1970.

[6] Correia, H.M.G. and Ramos, M.M.D.: Quantum modelling of poly(vinylidene fluoride). Computational Materials Science 33 (1-3), 224-229 (2005). 
[7] Ramos, M.M.D., Correia, H.M.G., and Lanceros-Mendez, S.: Atomistic modelling of processes involved in poling of PVDF. Computational Materials Science 33 (1-3), 230$236(2005)$.

[8] Hilczer, B. and Kulek, J.: The effect of dielectric heterogeneity on the pyroelectric response of PVDF. IEEE Transactions on Dielectrics and Electrical Insulation 5 (1), 45-50 (1998). 